\title{
A Combined Inventory Routing and Game Theory Approach for a Real-World Distribution Problem
}

\author{
Manel Mateo ${ }^{1}$, El-Houssaine Aghezzaf ${ }^{2}$ and Pau Vinyes ${ }^{1}$ \\ ${ }^{1}$ Technological University of Catalonia, UPC, Spain (manel.mateo@upc.edu) \\ ${ }^{2}$ Faculty of Engineering, Ghent University, UGent, Belgium (elhoussaine.aghezzaf@ugent.be)
}

\begin{abstract}
In this paper, we develop a solution approach combining inventory routing and game theory and discuss it application to solve a real-world distribution and logistics problem. The problem consists of developing a distribution plan to replenish inventory at the involved sales-points and then clustering these sales-points into groups willing to cooperate and share a part of their stocks. The distribution and clustering strategies should be developed in such a way so as to minimize distribution and inventory costs of the system. The cooperation inside each cluster increases the potential inventory to which each member of this cluster has access. This enhances, in turn, the service level of each sales-point. The approach is implemented for a real-life case and the obtained results are reported and discussed in this paper.
\end{abstract}

Keywords: Inventory Routing, Game Theory, Case Study

\section{INTRODUTCION}

The inventory routing problem (IRP) is an underlying optimization model for the vendor managed inventory strategy involving integration and coordination of the inventory management and vehicle routing components in a logistical chain. More precisely, the IRP is concerned with the distribution of some product from a given facility to a set of sales-points over a given planning horizon. It is assumed that each sales-point uses or consumes the product at some given rate, which may or may not be constant, and can maintain an inventory of the product up to some given level. This inventory is called local inventory. A fleet of vehicles of same or different capacities are available for the distribution of the product. The main objective is to minimize the distribution costs during the planning period without causing any stockout at any of the sales-points.

Game theory can be viewed as a multi-agent decision-making problem. This means that there are many decision makers contending for limited rewards/payoffs. These decision-makers have to make certain decisions on which their payoff depends, and have to follow certain rules while making these decisions. Each decision-maker is supposed to behave rationally. Under the current problem sitting, agents or decision-makers are managers of sales-points who must decide how much local inventory to carry. Each sales-point wishes to carry an as low as possible amount of local inventory, and at the same time is reluctant to miss any potential sale. A possibility to cooperate is then offered to these sales-points, they can therefore trade the product amongst them at some extra fair price. Thus, a sales-point which at some point in time realizes that it has an excess inventory may want to sell that surplus to other sales-points, from the same cluster, experiencing larger demands than expected. Consequently, the game consists of determining clusters of sales-points which are willing to cooperate, a fair trade-price, and inventory quantities to be carried by each sales-point to realize an overall win-win situation with a minimal total costs. Game theory is used at this level.

\section{THE CASE STUDY}

The case discussed in this paper is that of a leading retailer of wine and liquor in United Kingdom. Its business model is based on franchising with more than 2000 stores scattered all over the country. Although a large amount of wine that the company distributes is sold in supermarkets, these specific stores represent approximately $30 \%$ of the sales. This percentage is further subdivided as follows: $14 \%$ is distributed through the sector of retail chains (in which the company has a share of $30 \%), 10 \%$ is distributed to independent groceries, and the rest is distributed to independent sales-points specialized in wine products. Ultimately, the company sells as much as 3.5 to $4.2 \%$ of the total amount of wine sold in United Kingdom.

This study focuses on a special product developed in Spain for the company. We analysed the distribution of this product from the local warehouse of the company in the United Kingdom to each one of the franchised stores. Data in this study is modified to preserve the privacy for the company; however the figures remain very close to reality. The special product is distributed to some 116 sales-points scattered in some 49 cities. For the distribution of the product a fleet of exclusive vehicles is used. This fleet is composed of three vehicle categories: vans of 1.5 tones, trucks of 3 tones and then trucks of up to 10 tones.

978-1-4244-4136-5/09/\$25.00 @2009 IEEE 
A van of 1.5 tones has a capacity of two pallets and its corresponding fixed and variable costs are respectively $167.21 € /$ day (or $18.58 € / \mathrm{h}$ ) and $0.187 € / \mathrm{Km}$. Vans are usually used to serve sales-points with low demand. A truck of 3 tones has a capacity of 4 pallets and its corresponding fixed and variable costs are respectively $178.09 € /$ day (or $19.79 € / \mathrm{h}$ ) and $0.231 € / \mathrm{Km}$. Truck of 3 tones are used for sales-points at short-distances, with limited load and frequent trips, from the distribution centre. Trucks of up to 10 tones compose the largest category in the fleet. These vehicles differ only in length and their corresponding capacities increase by two pallets from one model to the next larger one. Therefore, the capacities go from 6 pallets, 2 more than the truck of 3 tones, up to the 12 pallets for the $6.2 \mathrm{~m}$ long trucks (longer trucks are considered for long distances). The fixed and variable costs for trucks of 10 tones are respectively $213.23 € /$ day (or $23.69 € / \mathrm{h}$ ) and $0.291 € / \mathrm{Km}$. To assign a cost to intermediate trucks (between 3 and 10 tones), a $5 \%$ on the fix cost and a $10 \%$ on the variable cost is discounted.

The standard pallet used throughout the paper is the British pallet. The pallet size is always the same $(1.2 \mathrm{~m} \times 1 \mathrm{~m})$. The $1.2 \mathrm{~m}$ side is used for the vehicle width (allowing a maximum of two pallets). The capacity in pallets of a truck is determined by its length and the $1 \mathrm{~m}$ side of the pallet. This capacity is thus given ( 2 pallets $x$ the length of the truck in meters). The largest vehicle carries 12 pallets.

\section{THE SOLUTION APPROACH}

The solution approach consists of developing a routing planning which allows each sales-point to be replenished and in the same time constructing clusters which will cooperate so that as to assure that less possible potential demand are lost. Game theory will be used to determine the opportunity cost of keeping inventory and sell it if necessary to any other. In the following paragraphs we describe the steps in developing such a solution.

\subsection{The Distribution Problem}

The main objective of the distribution problem is to optimize the logistical and inventory costs while assuring that all demand are satisfied. The underlying optimization model for this typical problem is known as the Inventory Routing Problem (IRP). The model tries to schedule supplies to each sales-point in such a way that will avoid causing stockouts and keeping the overall distribution and inventory cost as minimal as possible.

For this study, the IRP model proposed by Aghezzaf et al. (2006) is adopted. This model is a variant of the general IRP problem, where distribution is planned in a cyclical way from multiple distribution centres, denoted by $r_{k}(k=1, \ldots, p)$ to a set of sales-points $S(i=1, \ldots, n)$.
Each sales-point $i$ in $S$ has a demand rate $d_{i}$, given in units per hour. A fleet of homogeneous vehicles $V$ having capacity $\kappa$ are used to supply these sales-points.

\subsubsection{The mixed integer formulation of the problem}

In case of single depot, the formal mix integer formulation for the problem as is proposed in (Aghezzaf et al. 2006) is based on the following assumptions:

1. The time necessary for loading and unloading a vehicle is relatively small compared with travel times, and it is therefore neglected.

2. Inventory capacity at the sales-points is assumed to be large enough, so corresponding capacity constraints can be omitted from the model.

3. The travel costs are assumed to be proportional to the travel times, so we only use a parameter $\delta$ that indicates the cost per hour of travel.

Let $t_{i j}$ (in hours) denote the duration of the trip from sales-point $i \in S^{+}=S \cup\{r\}$ to sales-point $j \in S^{+}$. Also, let $\varphi_{i}$ and $\eta_{i}$ denote, respectively, the inventory fixed ordering and holding costs at sales-point $i$. The fixed operating and maintenance costs of vehicle $v \in V$ (in Euro per hour) is denoted by $\psi^{v}$. The solution of the problem consists of replenishing sales-points assigned to each vehicle $v \in V$ during related cycle time.

The model variables are:

- $\quad x_{i j}^{v}$ : A binary variable assuming 1 if the sales-point $j$ is served by the vehicle $v$ immediately after the sales-point $i$; and 0 otherwise.

- $\quad y^{v}$ : A binary variable assuming 1 if the vehicle $v$ is used; and 0 otherwise.

- $\quad z_{i j}^{v}$ : The sum of demand rates (units per hour) of remaining sales-points in a tour covered by vehicle $v$ when it travels to a sales-point $j$ immediately after it has served sales-point $i$. This quantity equals zero when the trip $(i, j)$ is not on any tour made by vehicle $v$.

- $T^{v}$ : The cycle time (in hours) of the multi-tour made by vehicle $v$. It must be strictly positive to avoid division by zero in the objective function.

The initial non-linear mixed integer formulation of the IRP:

IRP $_{\text {IP }}:$ Minimize

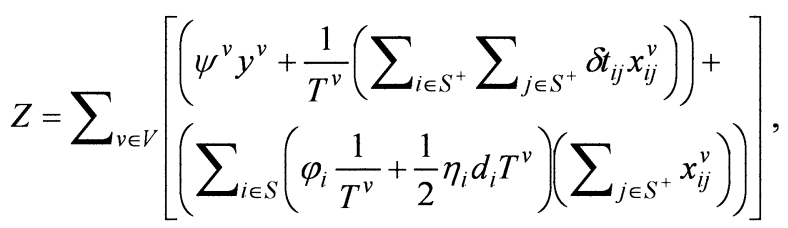


Subject to:

$$
\begin{aligned}
& \sum_{v \in V} \sum_{i \in S^{+}} x_{i j}^{v}=1, \quad \forall j \in S \\
& \sum_{i \in S^{+}} x_{i j}^{v}-\sum_{k \in S^{+}} x_{j k}^{v}=0, \quad \forall j \in S, v \in V \\
& \sum_{i \in S^{+}} \sum_{j \in S^{+}} t_{i j}^{v} x_{i j}^{v}-T^{v} \leq 0, \quad \forall v \in V \\
& \sum_{v \in V}\left(\sum_{i \in S^{+}} z_{i j}^{v}-\sum_{k \in S^{+}} z_{j k}^{v}\right)=d_{j}, \quad \forall j \in S \\
& x_{r j}^{v}-y^{v} \leq 0, \quad \forall v \in V, j \in S \\
& T^{v} z_{r j}^{v} \leq \kappa, \quad \forall j \in S, v \in V \\
& z_{i j}^{v}-\left(\sum_{k \in S} d_{k}\right) x_{i j}^{v} \leq 0, \quad \forall v \in V, i, j \in S^{+}
\end{aligned}
$$

The constraints (1) make sure that each sales-point is served by one and only one vehicle. Constraints (2) assure that a vehicle assigned to serve a sales-point will serve this point in and will leave to a next sales-point. The constraints (3) indicate that the cycle time of a vehicle should be greater than the total travel time necessary to complete the trip. It includes the duration of all tours made by the vehicle. The constraints (4) assure that the cumulated annual demand rates, per hour, of the remaining sales-points in the tour carried by the vehicle $v \in V$ serving the sales-point $j \in S$ is reduced by the demand rate $d_{j}$ when the vehicle leaves this sales-point. Constraints (5) make sure that a vehicle can only leave the distribution center $r$ to make a voyage if it is being used. The constraints (6), which are non-linear, are vehicle capacity restrictions. Constraints (7), finally, assure that $z_{i j}^{v}$ cannot carry any cumulated demand rates unless $x_{i j}^{v}$ equals 1 .

\subsubsection{An approximation algorithm}

In the following we summarise the approximation algorithm proposed in (Aghezzaf et al., 2006). This algorithm is based on a column generation process in which the subproblem generating multi-tours is solved heuristically. More specifically, the subproblem is solved using a heuristic which extends the savings-based heuristic used to solve Vehicle Routing Problems.

A multi-tour $v$ is defined by the binary matrix $X^{\prime \prime}$, the matrix $Z^{\prime}$ and the parameter $T^{v}$ such that $x_{i j}{ }^{\prime}=1$ if multi-tour $v$ contains a trip that visits the sales-point $j$ immediately after the sales-point $i$. The cost rate of the multi-tour can be computed as follows:

$$
\begin{aligned}
& K^{v}=\psi^{v}+\frac{1}{T^{v}}\left(\sum_{i \in S^{+}} \sum_{j \in S^{+}} \delta t_{i j} x_{i j}^{v}\right)+ \\
& \sum_{i \in S}\left(\varphi_{i} \frac{1}{T^{v}}+\frac{1}{2} \eta_{i} d_{i} T^{v}\right)\left(\sum_{j \in S^{+}} x_{i j}^{v}\right)
\end{aligned}
$$

Let $P V$ be the collection of all possible multi-tours $\left(X^{\prime \prime}\right.$, $\left.Z^{\prime}, T^{\prime}\right)$ satisfying required restrictions, then problem IRP problem can be reformulated as follows (called as IRP $\mathrm{MG}_{\mathrm{MG}}$ :

$$
\mathrm{IRP}_{\mathrm{MG}}: \quad \text { Minimize } \sum_{v \in P V} K^{v} w_{v}
$$

Subject to:

$$
\begin{gathered}
\sum_{v \in P V}\left(\sum_{i \in S+} x_{i, j}{ }^{v}\right) w_{v}=1, \text { for all } j \in S \\
w_{v} \in\{0,1\}, \quad \text { for all } v \in P V
\end{gathered}
$$

This new problem seeks the best combination of multi-tours $\left(w_{v}=1\right.$ if the multi-tour $v$ is selected, and 0 otherwise) which covers the sales-points while minimizing the costs. It is the master problem in the column generation process. Thus, an efficient procedure of generating columns is necessary to determine the multi-tours, so that the new variant of problem IRP can reach the nearly best solutions as fast as possible. The multi-tour generation procedure is summarized below:

1. Initially a list of multi-tours $P V$ is created with the $|S|$ 'basic' multi-tours, i.e. those that visit only one sales-point.

2. The linear programming relaxation of problem $I R P_{M G}$ is solved.

3. From the solution of the $L P$ relaxation of problem $\underline{\mathrm{IRP}}_{\mathrm{MG}}$, dual prices $\lambda_{j}$ for each $j \in S$, associated with constraints (9), are generated.

4. An attempt to determine a new feasible multi-tour with a negative reduced cost is launched. If such a multi-tour is found, it is added to the list $P V$ and the process returns to step 2 . If there is no such multi-tour, the $L P$ optimal solution is reached and the process moves to step 5 .

5. Problem $I R P_{M G}$ is solved (PV is the set of all feasible multi-tours which are generated) to provide the final solution.

To determine the feasible multi-tour with the least reduced cost, a multi-tour-generator subproblem $I R P_{S P}$ is solved with an extended savings-based heuristics (for more details see Aghezzaf et al., 2006).

\subsection{Application of Game Theory}

Game theory can be adapted to this distribution problem in order to maximize the sales opportunities of the company and to increase its revenues (Chan and Lee, 2005; Ghiani et al., 2004). As is already mentioned, the product that the company distributes comes from Spain and requires a long lead time to arrive to UK. Thus, when actual demand for the product is higher than expected and there is not enough stock to satisfy this extra demand, sales-points may be losing a great deal of non recoverable sales during the ordering lead-time. An alternative to avoid losing these sales opportunities is to 
hold an important stock to cover the demand peak, but this comes with an overstocking cost. This shows that there are extra relevant opportunity losses and/or overstocking costs which could be optimized with the help of Game Theory.

Game theory can be adapted to this distribution problem to maximize the sales opportunities of the company under variables demand rates. The basic idea is the creation of cooperative cells which will cooperate. A typical cooperative cell includes sales-points which are located within some delimited geographic area. Thus, different groups of sales-points will be created over the British territory and the cooperation within each group of sales-points can be seen as a local cooperative game. Therefore, a sales-point can decide to or not cooperate with other sales-points in the group. This fact, despite the simplification of the problem, leads to a practical and realistic solution. When a sales-point has no enough stock available and if the objective is to minimize the possible stockout duration, extra inventory of the closest sales-points could be a solution. Moreover, this fact is also coherent with the company culture, which wants to establish some cooperation between the sales points.

The cooperation mechanisms can be developed at different levels, according to the coverage of the demand peak. If a demand increase is experienced only in a neighbor or a village, the encouraged cooperation between sales-points can without difficulty take place. However, if a demand increase is experienced in a county or a region, all the sales-points will experience this demand increase and the cooperation will be difficult to establish. In this case the cooperation game should be established rather at the level of distribution warehouses. Therefore, although the model is intended for solving local problems, it can be extended to wider situations. Once again in the definition of the problem, the main parameter is the number of bottles required by a "cooperative cell" to solve possible stock problems of its sales-points $\boldsymbol{G}=\{1, \ldots, m\}$. Let us denote by $\boldsymbol{f}$ the total number of required bottles. The set of service providers, the sales-points of the cooperative cell, are the players and are denoted by $N=\{1, \ldots, n\}$. The offer of services (offer profile $O P_{i}$ ) by each one of the players (sales-points) $i \in N$ is the number of bottles that each sales-point is willing to hand over. This value depends on the dimension of the sales-point and the forecasted demand. Therefore, there is a cooperative game problem on the demand, $\Omega=(\boldsymbol{N}, \boldsymbol{G}, \boldsymbol{O P}, \boldsymbol{f})$, for each group of sales-points (initial points).

In order to provide an incentive to the sales-points to cooperation, the agents may come to an agreement that the bottles intended for a collective usage will be sold at higher price, and that the third of the total benefit is granted to the sales-point that handed over the bottle. Based of this agreement, the profit for each possible coalition can be computed. Therefore, the process start with each sales-point considered as an isolated coalition, with a zero profit, as no additional bottle can be sold if the sales-point is alone. Then the coalitions of two, three sales-points and so on are investigated, to determine the optimal coalition.

The procedure works as follows: First, the total number of bottles that can be supplied by all sales-points in a cell is computed. Then, the number of bottles that can be distributed (lower value between the supply and the demand) is determined. If the supply is greater than the demand, the demand will be distributed. Otherwise, if the demand is greater than the supply, the number of bottles is limited to the amount that can be supplied. An additional number of bottles can be sold, as a result of the existence of the cooperative cell, generating an extra common profit. This profit is split in three parts, $2 / 3$ for the sales-point that receives and sells the bottle, and $1 / 3$ for the sales-point that supplied the bottle (Vinyes, 2007). Moreover, there is an additional term that is subtracted from this total profit. This can be better defined from a practical point of view. If a seller has one unique bottle at the end of a period, he will probably be reluctant to put it at the community's disposal, as he might be losing part of the profit ( $2 / 3$ to be exact) in case the sales-point itself gets the possibility of selling the bottle. On the other hand, if a sales-point has a larger amount of bottles at the end of the same period, he may be willing to put some or all of them at the community's disposal; as he might think that it might be difficult to sell all bottles. Thus, owing to this factor, the coalition with sales-points assignors and only one bottle in stock will provide a low global profit. This profit function (per period) will have the following pattern:

$$
\text { Coalition profit }=\left[1-\sum_{i}\left(\frac{1}{2.2+\text { Exchange }_{i}}\right)\right] \text { profit }
$$

In this preliminary analysis only coalition of up to four sales-points are considered. The profit is computed for any possible cooperation of four sales-points taken from one to one up to a single group of four, and the one that maximizes the extra profit is selected. Once this coalition found the Shapley's value (Shapley, 1953) of all included sales-points is determined. These values indicate the percentage or weight that each point has into the global coalition profit and, therefore, they will be the first to give or receive bottles.

\section{Simulation approach and related results}

To solve the complete problems (optimization and simulation), the Solver and Excel with some programmed macros in Visual Basic are used.

\subsection{Cooperative games results}

First, the tour construction minimizes the distribution cost. These tours were constructed using a multi-tour 
heuristic. The objective is to minimize the distribution costs with shorter cycles going through the distribution centre and serving the client more frequently. The stock control at each sales-point takes into consideration the forecasted demand and the frequency of the planned van or truck visits. Demand at each sales-point is assumed to be normally distribution. The average and standard deviation of this distribution is determined from the observed input values. In the simulation, the demand at each sales-point is generated per time period. The time period is decided among the members of the cooperative cell, in order to enable the communication of the player's demands and offers. In the simulation, it is fixed to a half labor-day (i.e. 4 hours).

In the current preliminary analysis, it is assumed that the demand of sales-points is the same and is constant. This fact is used to guarantee "fixed" tours. If the demands were assumed to be variable, the distribution problem would vary and the tours would differ each time. This is situation is more realistic and will be tackled in the future. However, we allow that all cycle times may vary, i.e. there are vehicles which last more than others to come back to the distribution centre. Since demands are assumed to follow a normal distribution with a constant average, theoretically after some time all the sales-points in a cell would have received and handed over the same number of bottles. We believe, though, that the tours with fixed and constant demands would perform better at a least cost. Therefore, the model is simplified long these lines as we consider that this would provide a valid solution for mid and long term.

The period considered for refreshing data is 4 hours, i.e. information will be updated each 4 hours. Initially, the cooperation has been established for groups of four sales-points, simulating the cooperation among them. In Table 1, one can see results of a small example. The demand at each sales-point is generated randomly from a normal distribution with a mean of 5 bottles and a standard deviation of 2.5 . Once the generated values for the demand and a forecasted stock for each sales-point are known, one bottle lack or excess is defined for each sales-point, and the Shapley values are generated. These values provide information about the potential extra profit that each sales-point contributes for any coalition to which it participates. In other words, if the profit per additional bottle sold and the mean profit generated by a sales-point are known, one can determine approximately which number of bottles the sales-point should contribute to the cell. These values are appended to the usual distribution, and are subtracted or added to the regular supply according to the requirements and relations yet established.

Table 1 shows that:

- The values of demand that are obtained from the normal distribution $N(5,2.5)$ and rounded to the nearest integer. Initial availability is 6 bottles at each sales-point.

- The total needed is 1 bottle and the total offered is 7 bottles; therefore, the interchange will be 1 .

- Based on computed Shapley values; sales-point 3 will receive the bottle from sales-point 1 .

\begin{tabular}{|c|c|c|c|c|}
\hline Sales-point & Demand & Stock & Needed & Offered \\
\hline S1 & 2 & 4 & 0 & 4 \\
\hline S2 & 4 & 2 & 0 & 2 \\
\hline S3 & 7 & -1 & 1 & 0 \\
\hline S4 & 5 & 1 & 0 & 1 \\
\hline Total & 18 & 6 & 1 & 7 \\
\hline
\end{tabular}

\begin{tabular}{|c|c|c|c|c|}
\hline Sales-point & Shapley & $\begin{array}{c}\text { Potential } \\
\text { profit (n) }\end{array}$ & $\begin{array}{c}\text { Potential } \\
\text { profit (o) }\end{array}$ & $\begin{array}{c}\text { Extra } \\
\text { flow }\end{array}$ \\
\hline S1 & 0.1081 & 0 & 0.1621 & -1 \\
\hline S2 & 0.0697 & 0 & 0.1045 & 0 \\
\hline S3 & 0.6036 & 0.9055 & 0 & +1 \\
\hline S4 & 0.0573 & 0 & 0.0859 & 0 \\
\hline
\end{tabular}

Table 1. Example of a cooperative cell when the needs are lower than offers.

On the other hand, Table 2 shows that:

- The total needed is 9 bottles and the total offered is 3 bottles; therefore, the interchange will be 3 .

- Based on computed Shapley values; sales-point 2 will receive one bottle from sales-point 3 , and sales-point 4 two bottles from sales-point 1 .

\begin{tabular}{|c|c|c|c|c|}
\hline Sales-point & Demand & Stock & Needed & Offered \\
\hline S1 & 4 & 2 & 0 & 2 \\
\hline S2 & 10 & -4 & 4 & 0 \\
\hline S3 & 5 & 1 & 0 & 1 \\
\hline S4 & 11 & -5 & 5 & 0 \\
\hline Total & 30 & -6 & 9 & 3 \\
\hline
\end{tabular}

\begin{tabular}{|c|c|c|c|c|}
\hline Sales-point & Shapley & $\begin{array}{c}\text { Potential } \\
\text { profit (n) }\end{array}$ & $\begin{array}{c}\text { Potential } \\
\text { profit (o) }\end{array}$ & $\begin{array}{c}\text { Extra } \\
\text { flow }\end{array}$ \\
\hline S1 & 1.3048 & 0 & 1.9572 & -2 \\
\hline S2 & 0.4343 & 0.6514 & 0 & +1 \\
\hline S3 & 0.5885 & 0 & 0.8828 & -1 \\
\hline S4 & 0.4343 & 0.6514 & 0 & +2 \\
\hline
\end{tabular}

Table 2. Example of a cooperative cell when the needs are higher than offers.

\subsection{Results of the tour-generation problem}

We consider 3 working days with a single shift of 8 hours, the periods are defined to be of 4 hours, and 6 periods are considered for the simulation. During this time, nearly 400 additional bottles have been sold in a subset of 116 sales-points. This amount represents $11 \%$ of higher sales with respect to the quantity without cooperation between sales-points. With extrapolation to the whole set of sales-points, and the working days per year (the sales-points of the company are closed during 
very few days each year; we can suppose 365 labour days per year), we obtain the results below (Table 3 ):

\begin{tabular}{|l|r|}
\hline $\begin{array}{l}\text { Sales of the set of the group } \\
\text { (extrapolation to } 1000 \text { sales-points): }\end{array}$ & $31,439.66 €$ \\
\hline $\begin{array}{l}\text { Year sales of the group (365 labour } \\
\text { days/year): }\end{array}$ & $3,825,158.05 €$ \\
\hline Year sales without cells: & $3,422,399.43 €$ \\
\hline Profit: & $402,758.62 €$ \\
\hline
\end{tabular}

Table 3. Extra profit due to cooperative cells.

Clearly the cooperation established between these subsets of sales-points helps achieve the sales objectives fixed by the company. Therefore, its implementation is recommended.

In order to determine the ideal routes or tours, the algorithm can be used for different kinds of vehicles and their capacities. Thus, once the routes selected, it is necessary to decide how many vehicles will be used to supply each sales-point with the desired frequency. Taking into account these values the global cost of the distribution on the geographical zone is calculated. To answer this question, let us first observe that a demand of 575 bottles for all sales-points each 4 hours is very small with respect to the system's capacity. It is small as all the vehicles of the company can supply the demand of the sales-points in the area using less than $50 \%$ of their capacity. The van, the smallest mean of transport, can transport 1800 bottles. Therefore, the solution is made of tours which can be obtained by a TSP heuristic. For the obtained solution, the cycle time is approximately 74 real hours (which are over 9 working days). As the capacity is not an active constraint, the van is chosen as it is the cheapest transportation mean. This fact means that to supply the sales-points each 4 hours a fleet of 19 vehicles with the above cited characteristics are necessary. If the cost of a cycle is $480.45 €$, the annual cost would be $356,000 €$.

\section{CONCLUSION}

This work has shown that Game Theory, and cooperative games in particular, is a very useful tool to improve efficiency of some supply chains. The real case studied in this paper shows clearly that a better solution resulting in an extra profit can be achieved with the help of Game Theory. Although the objective of each sales-point is to maximize its own profit, cooperation will certainly have no negative effect on their respective profits. Instead, it helps them realize extra sales and it allows the company to realize extra profit from these partnerships. Actually, businesses that are working under franchises could use this approach and cooperate in order to avoid disruption of the production, due to lack of raw materials, and to minimize the related logistical costs.

The routing design has been established using a heuristic, and takes into account the cooperation features. This design is essential to make cooperative cells possible. However, some improvements are still needed. In this preliminary analysis, the cooperative cells are composed of four members. It would be interesting to analyze efficiency of cells as a function of the number of sales-point members, taking into account the distance between these sales-points. Also, the current study assumes constant demand for all sales-points. We are planning to considerer varying demands generated by the periodic coalitions of the sales-points. Finally, it would be interesting to run a simulation in the time-scale, to determine for each sales-point the demand for the product at which it stabilizes, and then determine what the benefit could be on average.

\section{REFERENCES}

[1] Aghezzaf, E.-H., B. Raa, H. Van Landeghem, "Modeling inventory routing problems in supply chains of high consumption products", European Journal of Operational Research, 169, pp 1048-1063, 2006.

[2] Chan, C., H. Lee, "Successful Strategies in Supply Chain Management", IGI Global, Hong Kong, 2005.

[3] Ghiani, G., G. Laporte, R. Musmanno, "Introduction to logistics systems planning and control", Hoboken, NJ, USA: J. Wiley, 2004.

[4] Raa, B., E.-H., Aghezzaf, "A practical solution approach for the cyclic inventory routing problem", European Journal of Operational Research. Volume 192, Issue 2, pp. 429-441, 2009.

[5] Shapley, L. S., "A value for n-person games. In Contributions to the Theory of Games II.", A.W. Tucker and R.D. Luce (eds.), Princeton University Press, 307-317, 1953.

[6] Vinyes, P., "Anàlisis de la cadena de subministre d'una cadena de franquícies basada en la teoria de jocs.", Master thesis ETSEIB (UPC), 2007. 